\title{
A chemical approach for programmable protein outputs based on engineered cell interactions
}

\author{
Daniel A. Jacome ${ }^{1}$, Justin D. Northrup ${ }^{1,2,3}$, Andrew J. Ruff ${ }^{1}$, \\ Sean W. Reilly ${ }^{4}$, Iris K. Lee ${ }^{1,5}$, Gabrielle S. Blizard ${ }^{2}$, and Mark A. Sellmyer ${ }^{1,2, *}$
}

1. Department of Radiology, University of Pennsylvania, Philadelphia, PA 19104

2. Department of Biochemistry and Biophysics, University of Pennsylvania, Philadelphia, PA 19104

3. Institute for Translational Medicine and Therapeutics, University of Pennsylvania, Philadelphia, PA 19104

4. Department of Process Research and Development, Merck Research Laboratories, Kenilworth, NJ 07033

5. Department of Bioengineering, University of Pennsylvania, Philadelphia, PA 19104

* Correspondence should be addressed to:

M.A.S. (mark.sellmyer@pennmedicine.upenn.edu)

https://orcid.org/0000-0002-1407-1905

Department of Radiology and Biochemistry \& Biophysics

Perelman School of Medicine at the University of Pennsylvania

813A Stellar-Chance Labs

422 Curie Boulevard

Philadelphia PA

19104-6059

Phone: 215-573-3212

\section{Supporting Information}




\section{Methods:}

\section{Synthesis of pNZ-TMP}

$1 \mathrm{~g}$ of Trimethoprim was reacted with $916 \mathrm{mg}$ para-nitrobenzyl chloroformate (1.25 equiv.) and $438 \mu \mathrm{L}$ triethylamine (1.25 equiv.) in dimethylformamide overnight with stirring at room temperature. The reaction started cloudy, became clear after 5 minutes (yellowish), and formed a precipitate after the overnight reaction. The reaction mixture was diluted with three times the reaction volume of ethyl acetate, and washed three times with saturated ammonium chloride. During the washes, a yellow solid precipitated, which was collected with a Buechner funnel, and was left to dry overnight. The solid was purified by reverse-phase HPLC (5-95\% acetonitrile in water, $0.1 \%$ TFA) to give a white powder (30\% purified yield).

${ }^{1} \mathrm{H}, 400 \mathrm{MHz}, \mathrm{DMSO}-\mathrm{D}_{6}(1 \% \mathrm{TFA}): 3.65(3 \mathrm{H}, \mathrm{s}), 3.72(2 \mathrm{H}, \mathrm{s}) 3.75(6 \mathrm{H}, \mathrm{s}), 5.44(2 \mathrm{H}, \mathrm{s}), 6.63(2 \mathrm{H}, \mathrm{s})$, $7.06(1 \mathrm{H}, \mathrm{s}), 7.72(2 \mathrm{H}, \mathrm{d}, J=8.5), 8.28(2 \mathrm{H}, \mathrm{d}, J=8.4), 8.36(1 \mathrm{H}, \mathrm{s}), 8.85(1 \mathrm{H}, \mathrm{s}), 12.12(1 \mathrm{H}, \mathrm{s}) ;{ }^{13} \mathrm{C}$, $100 \mathrm{MHz}$, DMSO-D $(1 \%$ TFA): 32.7, 56.4, 60.4, 66.7, 106.9, 111.9, 114.4, 114.7, 117.7, 120.6, $124.1,129.1,132.5,136.8,140.4,143.5,147.8,150.1,153.5,154.0,158.2,158.6,158.9,159.3$, 164.3

\section{Tissue Culture/Cell-Line Generation}

HCT-116 (ATCC ${ }^{\oplus}$ CCL-247 $^{\text {TM }}$ ) and HEK-293T (ATCC ${ }^{\circledR}$ CRL-3216 $^{\text {TM }}$ ) wild-type (WT) cells were ordered from American Type Culture Collection (ATCC) and cultured using Dulbecco's Modified Eagle Medium (DMEM) with $10 \%$ fetal bovine serum (FBS) and $1 \%$ penicillin/streptomycin (P/S).

Cloning:

I. Receiver:

a. Luciferase and YFP: pBMN-I-GFP plasmid (\#1736) was acquired from Addgene. Synthesis of thermostable Luciferase-DD-DHFR construct was ordered through Genewiz. The construct was designed based on Tisi et al. (2002) with four point mutations: T214A, 1232A, F295L and E354K. The construct and destination vector were cut with $\mathrm{BamHI}$ and $\mathrm{Xhol}$ restriction enzymes, purified by gel electrophoresis, and ligated overnight resulting in the pBMN-tsLuc-DD-DHFR-IGFP plasmid. The fluorescent reporter plasmid was ordered from Addgene (\#29326).

b. IL-2: pTRPE-I-mCherry plasmid was acquired from a collaborating laboratory. Interleukin-2 fused to destabilized DHFR (IL-2-DD-DHFR) was synthesized and ordered via GenScript. The construct and destination vector were cut with $\mathrm{Xbal}$ and Sall restriction enzymes, purified by gel electrophoresis, and ligated overnight resulting in the pTRPE IL-2-DD-DHFR-I-mCherry plasmid.

II. Activator: pBMN-HA-NTR-I-GFP was prepared in a similar fashion with a nitroreductase (NTR) construct coming from pGL4.26-SS-352 found on Addgene (\#68791) 


\section{Retroviral Transduction}

Phoenix $^{\mathrm{TM}}$ Ampho cells (ATCC ${ }^{\oplus} \mathrm{CRL}-3213^{\mathrm{TM}}$ ) were cultured using DMEM with $10 \% \mathrm{FBS}$ and $1 \% \mathrm{P} / \mathrm{S}$. The cells were transfected with either pBMN-tsLuc-DD-DHFR-I-GFP or pBMN-HA-NTR-I-GFP plasmids using Lipofectamine 2000. Gene expression of GFP was checked after 24 hours with a fluorescence microscope. 48 hours post-transfection, the media from the cells was collected and filtered through a $0.45 \mu \mathrm{m}$ filter. Viral particles in the clarified media were then concentrated using a 100-kDa centrifugal filter concentrator (Millipore Sigma UFC910024). The concentrated viral particles were pipetted onto HCT-116 WT or HEK-293 WT cells and incubated for 5 hours before replacing with complete media. GFP positive cells were sorted by FACS.

\section{Lentiviral Transduction}

HEK-293T/17 cells (ATCC ${ }^{\circledR}$ CRL-11268 ${ }^{\text {TM }}$ ) were cultured using DMEM with $10 \%$ FBS without antibiotics. The cells were transfected with pTRPE IL-2-DD I-mCherry and second-generation packaging plasmids psPax2 and pMD2.G at a ratio of 4:3:2 by mass. 48 hours post-transfection, the supernatant from the cells was collected and filtered through a $0.45 \mu \mathrm{m}$ filter. Viral particles in the clarified media were then concentrated using a 100-kDa centrifugal filter concentrator (Millipore Sigma UFC910024). The concentrated viral particles were pipetted onto HCT-116 WT and incubated for 6 hours before replacing with fresh media. mCherry positive cells were sorted by FACS.

\section{Luminescence Measurements}

All assays were conducted by diluting stock D-luciferin $(15 \mathrm{mg} / \mathrm{mL})$ to a final concentration of $0.15 \mathrm{mg} / \mathrm{mL}$ in sample wells. The wells were read on a Tecan M2000 plate reader using a default luminescence script and $600 \mathrm{~ms}$ integration time.

\section{ELISA Experiments}

All assays were conducted using Abcam Human IL-2 ELISA (ab174444) according to manufacturer protocol.

\section{TMP and pNZ-TMP Dose Response}

Luminescence: HCT-116 tsLuc-DD-DHFR (receiver) cells were plated on a black, clear-bottom 96well plate at a density of 50,000 cells per well in $100 \mu \mathrm{L}$ of media. $5 \mathrm{mM}$ TMP and pNZ-TMP DMSO stock solutions were diluted 1:500 in complete media and then serially diluted 1:3 on a separate 96-well plate. The diluted drugs were added to reporter cells, and drug-free media was used as a control. The cells were incubated for 24 hours before luminescence was measured. The experiment was repeated three times in total.

Fluorescence: TMP or pNZ-TMP was serially diluted in 12 well plates in a volume of $500 \mu \mathrm{L}$. HCT116 YFP-DD cells were then plated onto the same 12 -well plates at a density of 400,000 cells per well in $500 \mu \mathrm{L}$ resulting a final volume of $1 \mathrm{~mL}$. The cells incubated with drugs for 24 hours before being trypsinized and analyzed via flow cytometry to determine YFP-DD expression. The experiment was repeated three times total. 
IL-2: HCT-116 IL-2-DD cells were plated in a clear 96-well plate at a density of 40,000 cells per well in $100 \mu \mathrm{L}$ of media. 5mM TMP and pNZ-TMP DMSO stock solutions were diluted 1:500 in complete media and then serially diluted $1: 3$ on a separate 96 -well plate. The diluted drugs were added to reporter cells, and drug-free media was used as a control. The cells were incubated for 24 hours before IL-2 was measured via ELISA. The experiment was repeated three times in total.

\section{Time Course (Flow Cytometry)}

HCT-116 YFP-DD-eDHFR cells were plated onto 6 -well plates at $1^{6}$ cells per well. TMP or pNZTMP was added to a final concentration of $2.5 \mu \mathrm{M}$ and incubated for $0,3,6,9$, or 24 hours. Cells were trypsinized and analyzed via flow cytometry to determine YFP-DD expression. The experiment was repeated three times in total.

\section{Western Blot}

HEK-293T WT and activator cells were lysed with RIPA buffer and protease inhibitor cocktail. Lysates were heated to $95^{\circ} \mathrm{C}$ in laemmli buffer and loaded onto NuPage 3-8\% Tris-Acetate gel with SeeBlue Plus 2 reagent and run in SDS running buffer. Peptides were transferred using an Invitrogen iBlot 2 system. Blots were placed in blocking solution ( $5 \% \mathrm{BSA}$ ) then moved into primary antibody solution probing with mouse anti-HA antibody and mouse anti-tubulin antibody. The blot was then washed twice with PBS and placed into secondary antibody solution before washing twice and detecting with Amersham ECL Prime Western Blotting Detection Reagents.

\section{Media Transfer}

Luminescence: HCT-116 WT or HCT-116 activator cells were plated on a clear 96-well plate at a density of 50,000 cells per well in $190 \mu \mathrm{L}$ of media. $2 \mu \mathrm{M}$ drug stocks of pNZ-TMP and TMP were prepared in complete media. The plated cells were dosed with $10 \mu \mathrm{L}$ of drug stock solution resulting in a final drug concentration of $100 \mathrm{nM}$ and incubated with activator cells for varying lengths of time of 0 to $\sim 7$ hours. $100 \mu \mathrm{L}$ of media from each of these conditions was removed and transferred to a separate black, clear bottom plate containing 50,000 HCT-116 receiver cells per well in $100 \mu \mathrm{L}$ media. After 24-hour incubation with receiver cells, the luminescence was measured on a plate reader. The experiment was repeated three times in total.

IL-2: HCT-116 WT or HCT-116 activator cells were plated on a 6 -well plate at $1.2 \times 10^{6}$ cells per well. pNZ-TMP or TMP was added to both cell types to a final concentration of $5 \mu \mathrm{M}$. After 48 hours of incubation, the media from a separate 6-well dish containing HCT-116 IL-2-DD cells at $0.5 \times 10^{6}$ cells was aspirated. The media that had been incubating with HCT-116 WT or HCT-116 activator cells was gently removed and transferred to the HCT-116 IL-2-DD cells and incubated for an additional 24 hours before IL-2 secretion was measured by ELISA. The experiment was repeated three times in total.

\section{Co-Culture of Activator and Receiver cells}

Luminescence: HCT-116 WT or HCT-116 activator cells were plated onto a black, clear bottom 96-well plate and serially diluted from a high concentration of $52.5 \times 10^{4}$ cells per well in a $1: 3$ 
fashion for seven total conditions of each cell line in $75 \mu \mathrm{L}$ of media. HCT-116 receiver cells were resuspended to $17.5 \times 10^{4}$ cells per $75 \mu \mathrm{L}$ and pipetted into the wells containing either HCT116 NTR or WT cells. Each condition received either pNZ-TMP, TMP, or no drug (media) at a final concentration of $50 \mathrm{nM}$ in a total volume of $200 \mu \mathrm{L}$ per well. The plates were incubated for 24 hours before luminescence was measured. The experiment was repeated three times total.

IL-2: HCT-116 WT or HCT-116 activator cells were plated onto a 6 -well plate at $2.4 \times 10^{5}$ cells per well. HCT-116 IL-2-DD cells were plated in the same wells at $6 \times 10^{4}$ cells per well. pNZ-TMP or TMP was added to a final concentration of $500 \mathrm{nM}$. No drug controls were present. The coculture incubated for 48 hours before IL-2 secretion was measured via ELISA.

\section{Co-Culture Kinetic Experiment}

HCT-116 WT or activator cells were plated at 20,000 cells per well in a black-walled, clearbottom 96-well plate. Additionally, 10,000 cells per well of HCT-116 receiver cells were plated in co-culture with either the WT or activator cells. Either pNZ-TMP or TMP was added to all conditions to achieve a final concentration of $50 \mathrm{nM}$ in each well and incubated for $1,2,4,7$, or 10 hours. The plate was then analyzed for luminescence using a plate reader. The experiment was repeated twice in total.

\section{Supplemental Figures}

\section{CB1954 Activation and Cytotoxicity}

The nitroreductase-activatable cytotoxic prodrug, CB1954, was acquired from Sigma (C2235) and prepared to $100 \mathrm{mM}$ stock solution in DMSO. HCT-116 WT or activator cells were plated at 20,000 cells per well in a black, clear-bottom, black-walled 96-well plate. CB1954 was diluted in media in a separate 96 -well plate and then transferred onto the cells resulting in a final concentration of $300 \mu \mathrm{M}$ at the highest dose and 1:3 dilutions thereafter. The cells were incubated in the presence of CB1954 for 72 hours and their viability was assessed using CellTiter Glo 2.0 (Promega G9241).

\section{Chemical Activation of pNZ-TMP}

Following a standard method for chemical reduction of para-nitrobenzyl carbamates (IsidroLlobet et al. 2005), 6 millimoles of Tin(II) chloride were dissolved in $1 \mathrm{~mL} \mathrm{DMF}$ with $16 \mu \mathrm{L}$ of $4 \mathrm{M}$ $\mathrm{HCl}$ in dioxane. $0.500 \mathrm{~mL}$ of this solution was added to $1 \mathrm{mg}$ of purified pNZ-TMP and reacted at RT for 1 hour with stirring. Reaction progress was monitored via HPLC-MS at 30- and 60-minute time points, showing complete reduction and cleavage of the pNZ group to provide Trimethoprim.

\section{Serum Stability of pNZ-TMP}

Diluted stock solutions of either TMP, pNZ-TMP, or DMSO control to a final concentration of $500 \mu \mathrm{M}$ in FBS and incubated at $37^{\circ} \mathrm{C}$ for 24 hours. The following day, $200 \mu \mathrm{L}$ of each solution was transferred to a $1.5-\mathrm{mL}$ tube. $1 \mathrm{~mL}$ of ice-cold acetonitrile was added to each sample to 
precipitate proteins. The solutions were centrifuged at $17,000 \mathrm{~g}$ for 15 minutes to sediment the proteins. The supernatant was filtered through a $0.45 \mu \mathrm{m}$ filter and the filtrate was analyzed via LC-MS. 


\section{Supplemental Figures:}

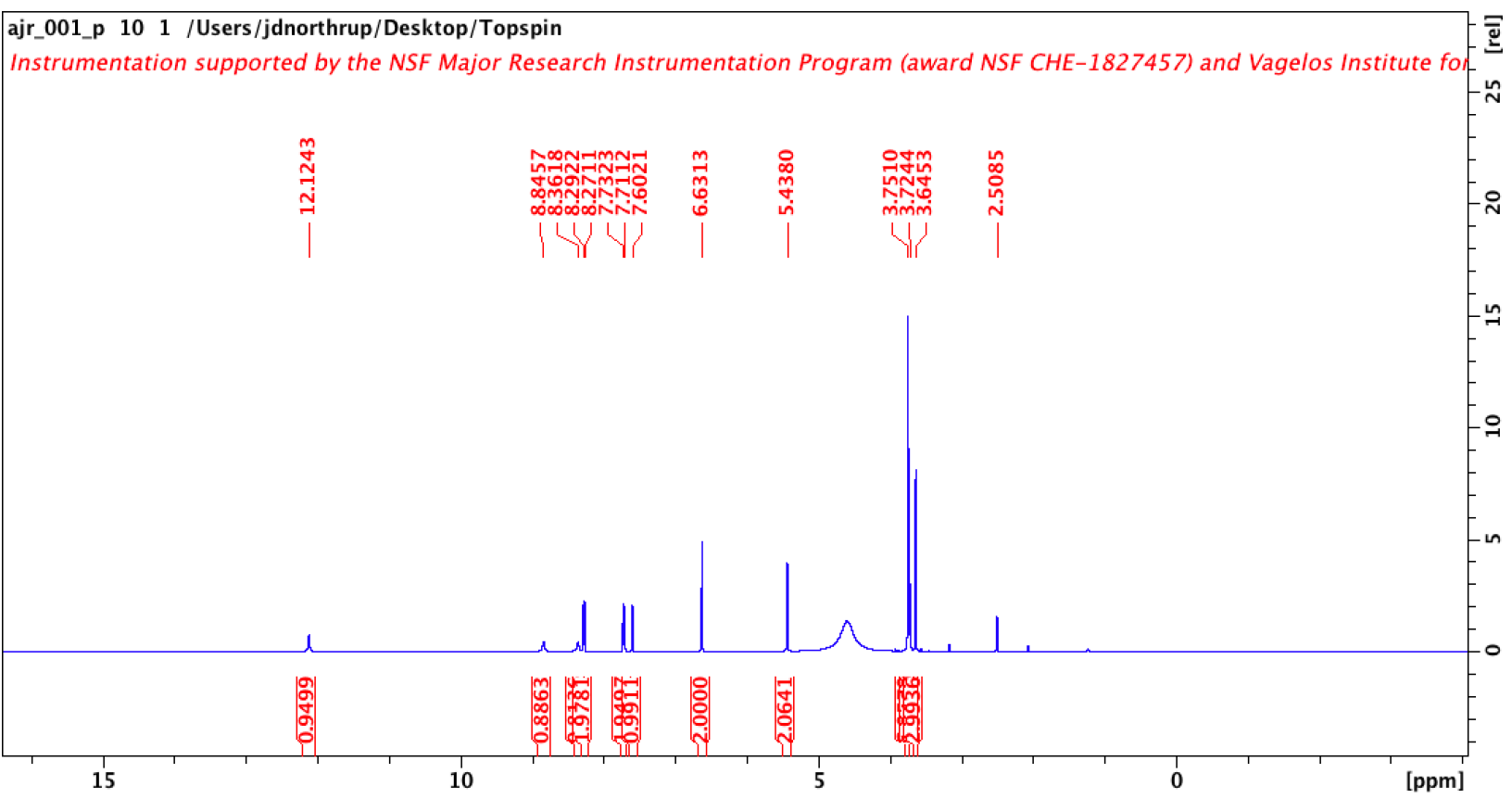

Supplemental Figure $1 .{ }^{1} \mathrm{H}-\mathrm{NMR}$ of pNZ-TMP

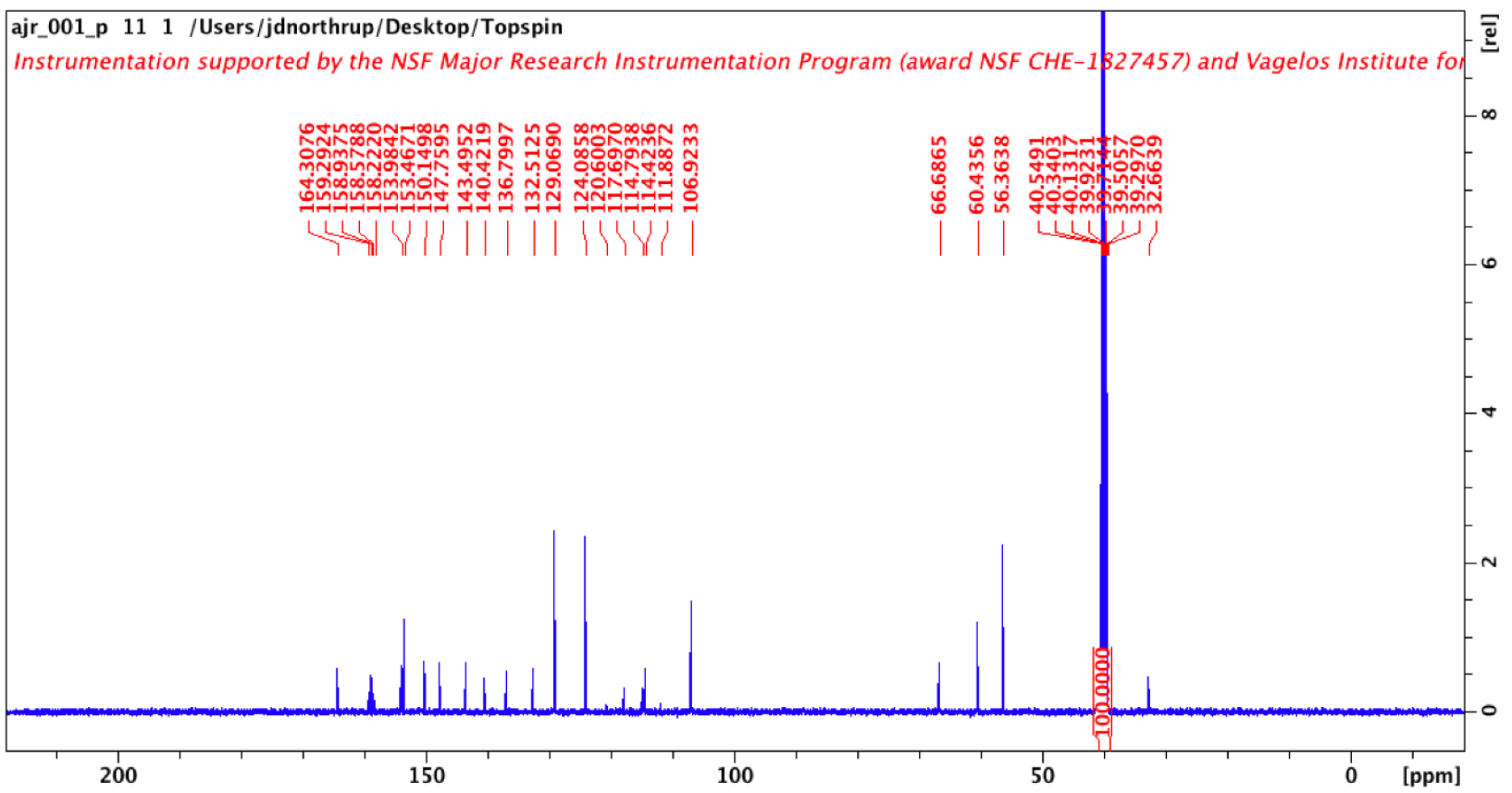

Supplemental Figure $2 .{ }^{13} \mathrm{C}-\mathrm{NMR}$ of pNZ-TMP 


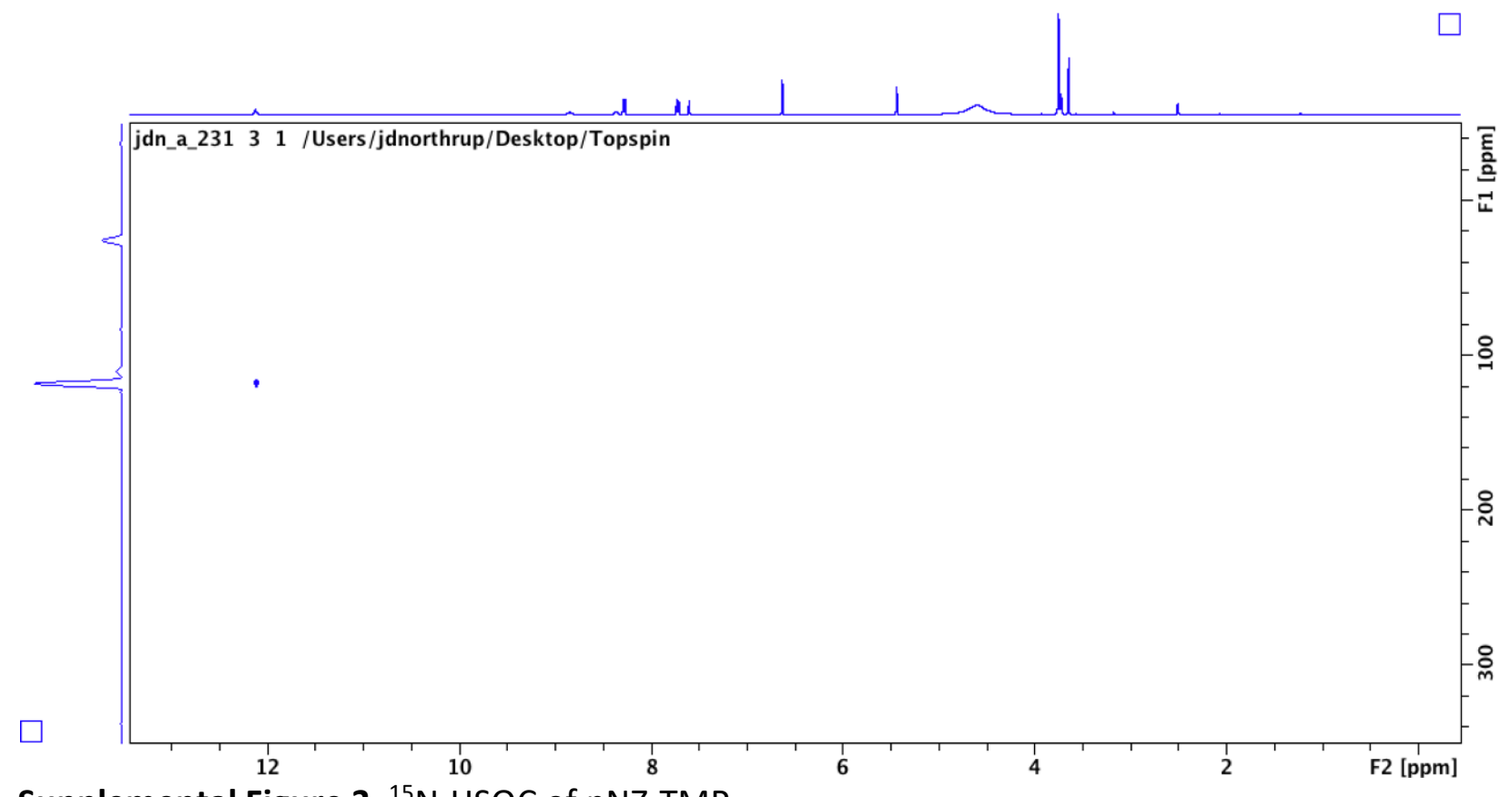

Supplemental Figure $3 .{ }^{15} \mathrm{~N}-\mathrm{HSQC}$ of pNZ-TMP 

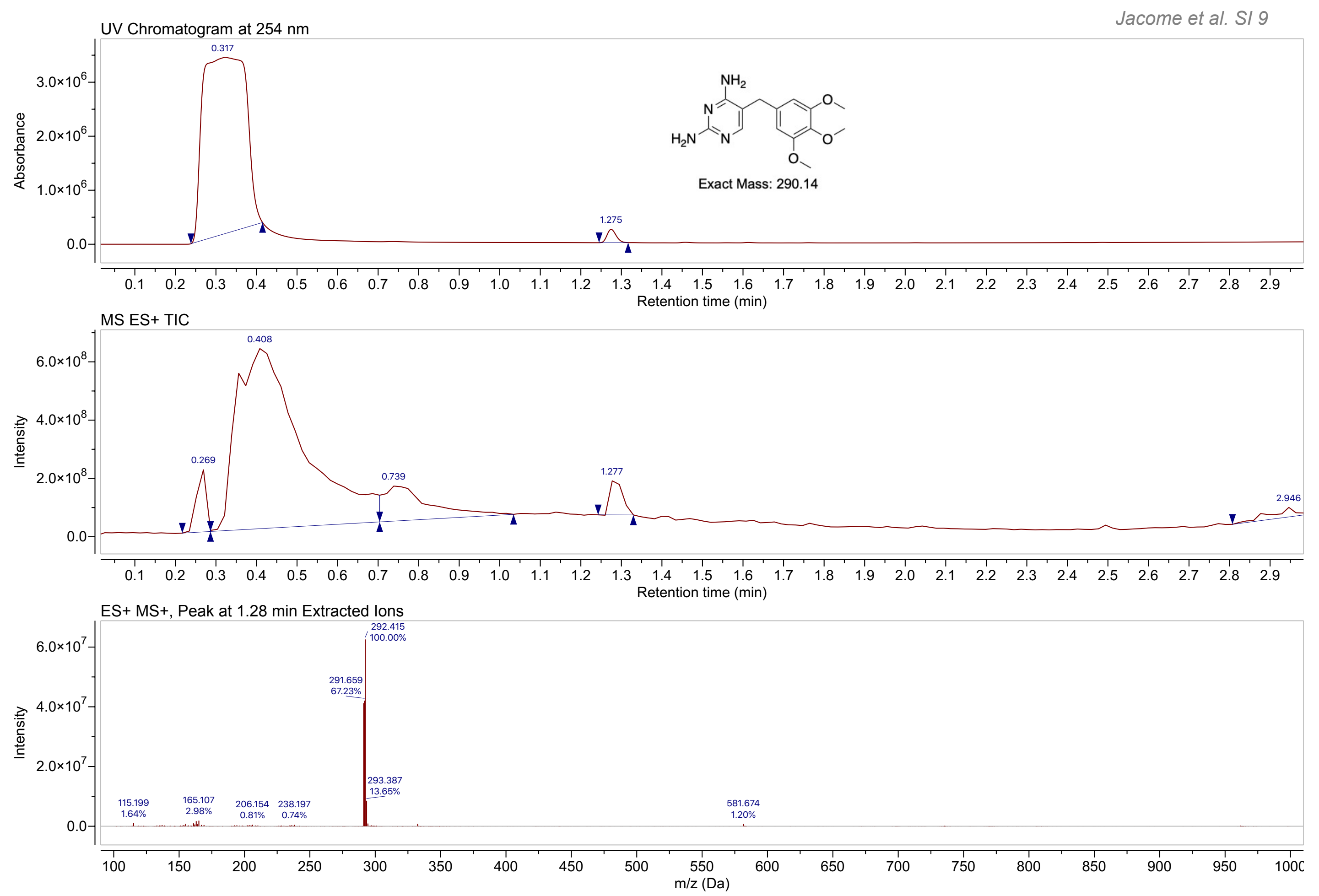

Supplemental Figure 4a. LC-MS trace of pNZ-TMP chemical reduction to TMP- 30 minutes 

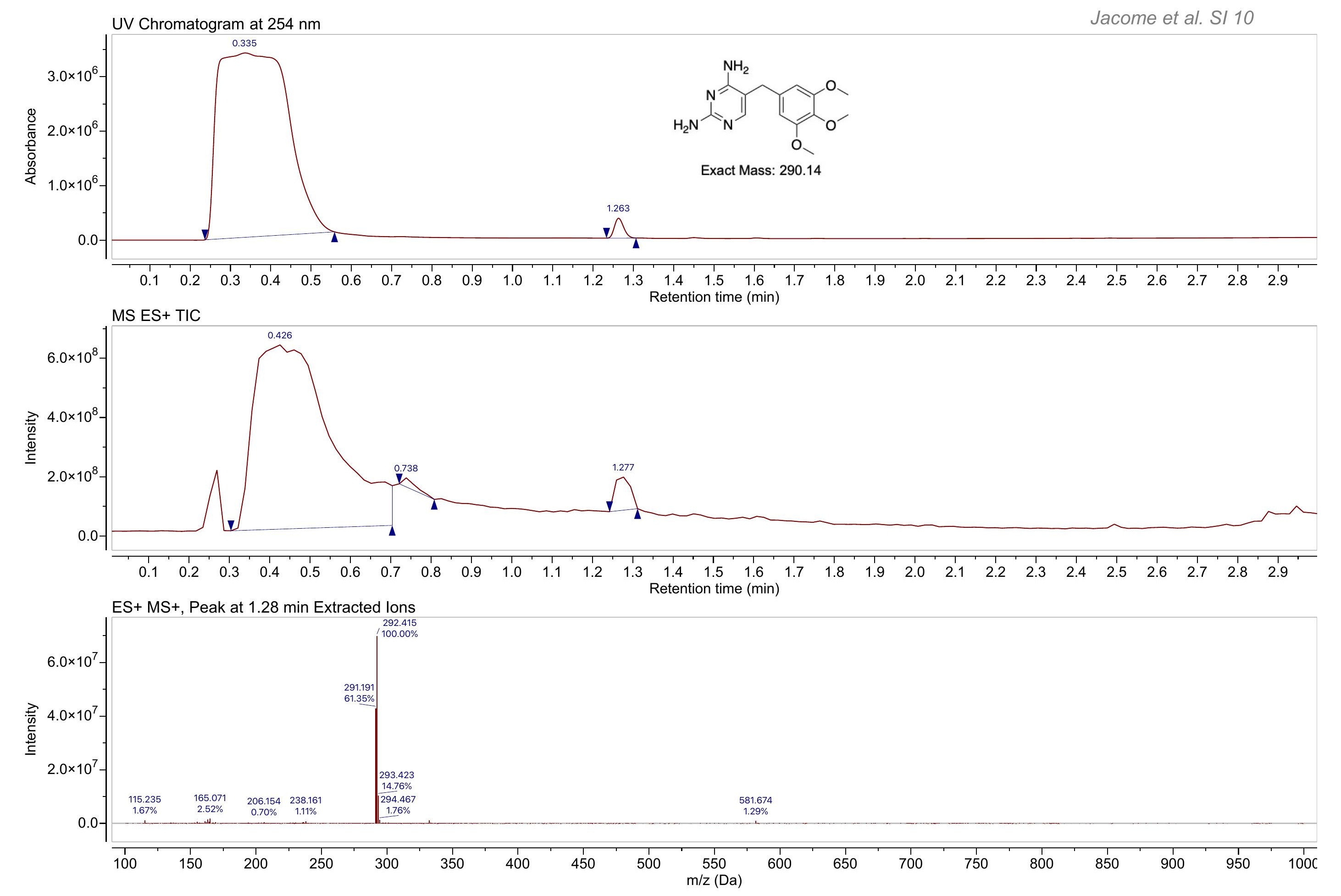

Supplemental Figure $4 \mathrm{~b}$. LC-MS trace of pNZ-TMP chemical reduction to TMP- 60 minutes 

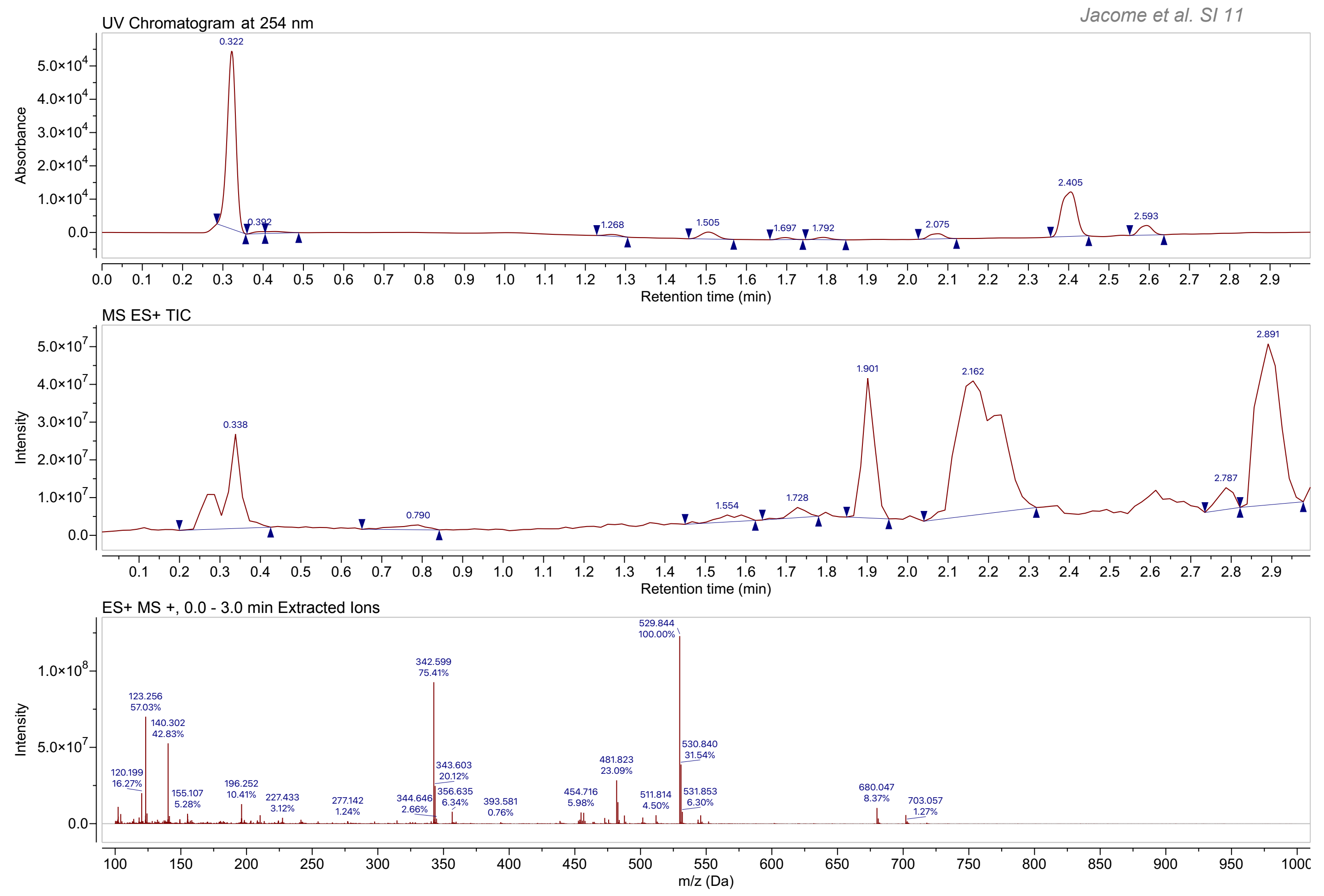

Supplemental Figure 5a. LC-MS trace of serum stability experiment- blank- $24 \mathrm{hrs}$ 

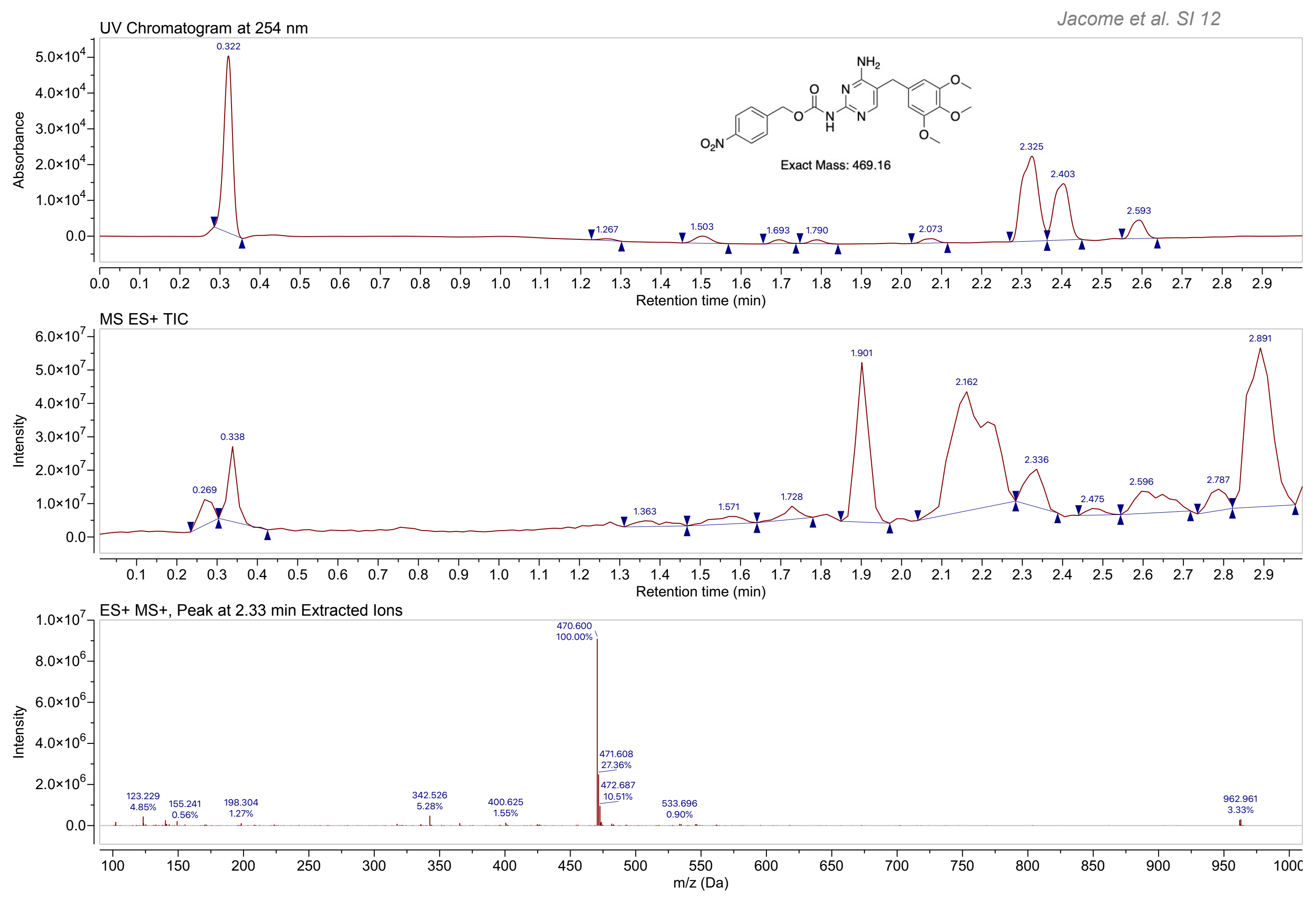

Supplemental Figure 5 b. LC-MS trace of serum stability experiment- pNZ-TMP-6 hrs 

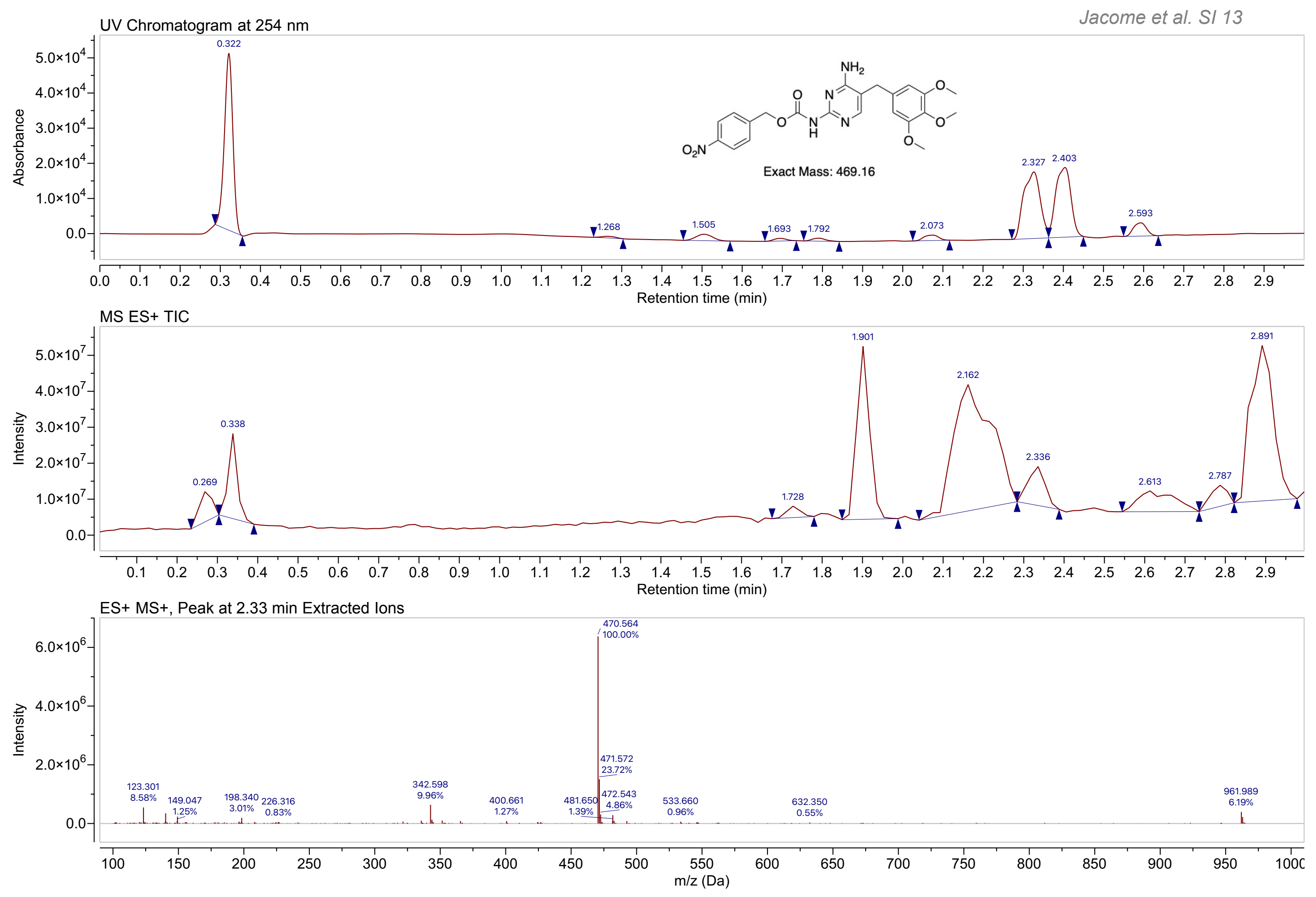

Supplemental Figure 5c. LC-MS trace of serum stability experiment- pNZ-TMP-24 hrs 

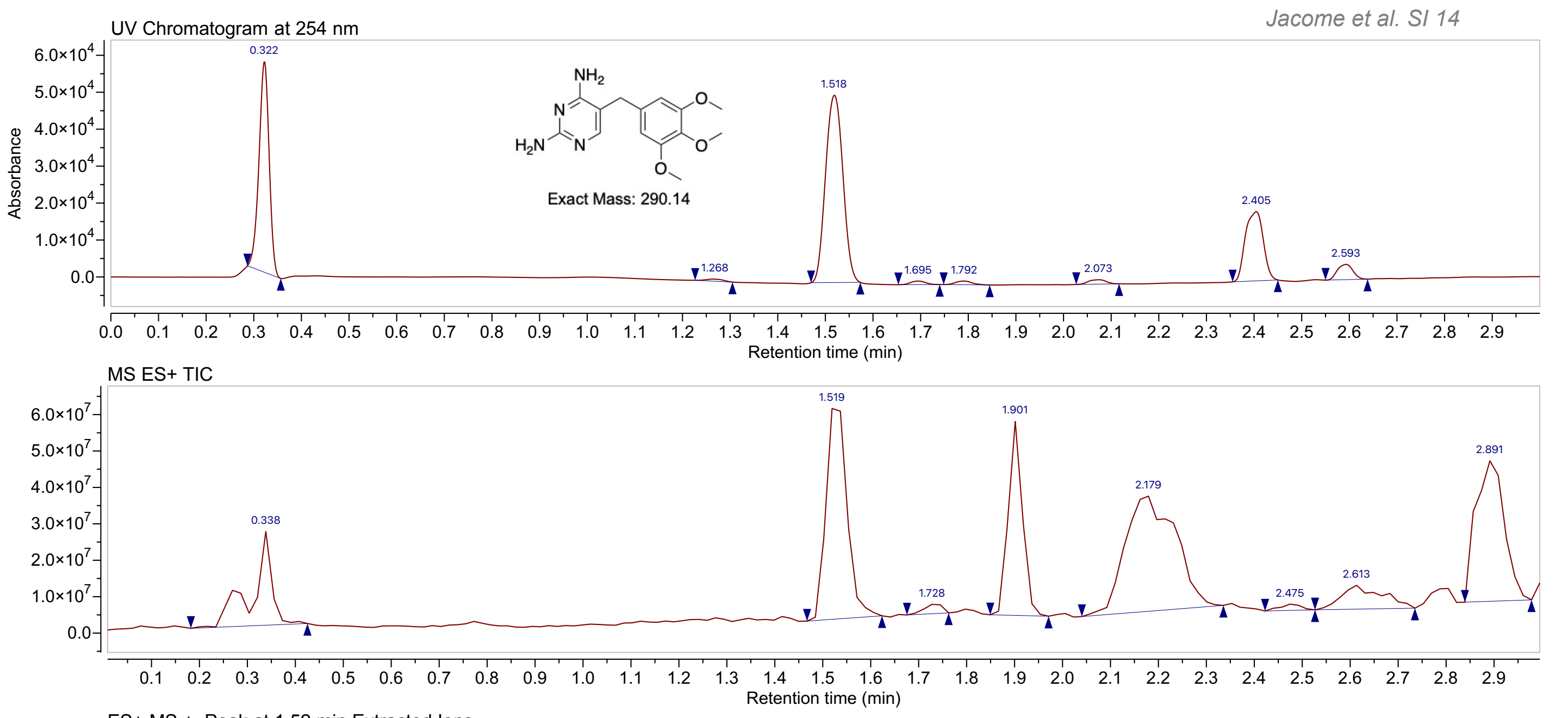

ES+ MS +, Peak at 1.52 min Extracted lons

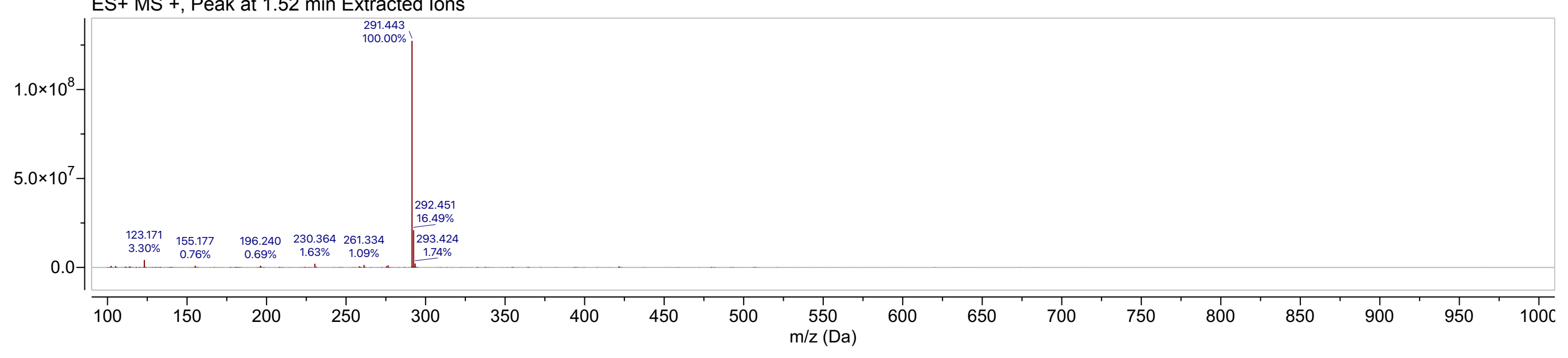

Supplemental Figure $5 \mathrm{~d}$. LC-MS trace of serum stability experiment- TMP-24 hrs 
CB1954 Activation and Cytotoxicity

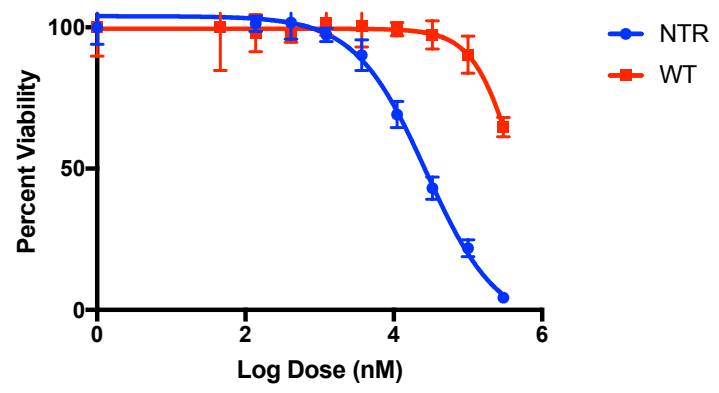

Supplemental Figure 6. Enhanced cytotoxicity of NTR labile prodrug in NTR bearing cells

\begin{tabular}{|r|r|r|r|r|r|r|}
\hline LogDose (pM) & \multicolumn{3}{|c|}{ TMP } & \multicolumn{3}{|c|}{ PNZ-TMP } \\
\hline 6.397940009 & 2785 & 2813 & 2878 & 537 & 539 & 540 \\
\hline 5.920818754 & 2432 & 2445 & 2437 & 485 & 487 & 489 \\
\hline 5.443697499 & 1938 & 1967 & 1935 & 376 & 372 & 374 \\
\hline 4.966576245 & 1096 & 1140 & 1166 & 260 & 268 & 259 \\
\hline 4.48945499 & 679 & 678 & 675 & 196 & 196 & 199 \\
\hline 4.012333735 & 373 & 362 & 396 & 156 & 155 & 160 \\
\hline 3.53521248 & 252 & 243 & 246 & 145 & 139 & 140 \\
\hline 3.058091226 & 172 & 167 & 170 & 127 & 130 & 135 \\
\hline 2.580969971 & 153 & 146 & 153 & 135 & 135 & 135 \\
\hline
\end{tabular}

Supplemental Figure 7. Raw mean fluorescence intensity data of technical replicates for YFP-DD dose response assay

\begin{tabular}{|r|r|r|r|r|r|r|}
\hline \multicolumn{1}{|c|}{ Hours } & \multicolumn{3}{|c|}{ TMP } & \multicolumn{3}{|c|}{ pNZ-TMP } \\
\hline $\mathbf{0}$ & 153 & 150 & 154 & 132 & 131 & 133 \\
\hline $\mathbf{1}$ & 312 & 305 & 306 & 133 & 129 & 130 \\
\hline $\mathbf{3}$ & 841 & 872 & 856 & 138 & 138 & 144 \\
\hline $\mathbf{6}$ & 1424 & 1465 & 1475 & 164 & 161 & 161 \\
\hline $\mathbf{9}$ & 1997 & 1917 & 1923 & 216 & 227 & 216 \\
\hline $\mathbf{2 4}$ & 3164 & 3167 & 3157 & 414 & 416 & 414 \\
\hline
\end{tabular}

Supplemental Figure 8. Raw mean fluorescence intensity data of technical replicates for YFP-DD time course assay 